\title{
Introduction
}

\section{The North/South Ireland Food Consumption Survey}

National dietary surveys are an important aspect of epidemiological research, nutrition monitoring, and the development of food and nutrition policies and programmes. Changing social circumstances and lifestyle factors, the growing choice of pre-packed convenience foods, the influences of immigrant foods, an increasing awareness of healthy eating messages as well as greater availability of low- and reduced-fat foods, and the emerging influence of food supplements and functional foods all suggest, even to the casual observer, that food consumption patterns in many countries are likely to have been substantially modified over the last decade. Some European countries, such as Sweden, Denmark, France and Italy, have recently re-commissioned surveys to keep abreast of changes in food consumption patterns whilst other European countries, such as The Netherlands, Germany and Great Britain, have instituted regular dietary surveys on a rolling basis as part of nutrition surveillance.

The first National Nutrition Survey in the Republic of Ireland was carried out by the Department of Health in $1948^{1}$ and the next was completed in $1988-89$ by the Irish Nutrition $\&$ Dietetic Institute ${ }^{2}$. Similar dietary information at the individual level was collected in Northern Ireland during $1986-87$ as part of the survey, Diet, Lifestyle and Health in Northern Ireland ${ }^{3}$. The Northern Ireland dietary survey used the same dietary intake methodology as the Dietary and Nutritional Survey of British Adults ${ }^{4}$ carried out in 1986-87, using the 7-day weighed record, whilst the Irish National Nutrition Survey ${ }^{2}$ utilised the diet history method with a photographic atlas of foods commonly eaten in Ireland. These two existing dietary databases, representing both parts of the island of Ireland, are clearly becoming dated and there was a need to update this information.

The North/South Ireland Food Consumption Survey (NSIFCS) is unique in that, for the first time, food consumption data representative of the population of Northern Ireland and the Republic of Ireland were collected at the same time using the same methodology. The survey was carried out during 1997 to 1999 by the Irish Universities Nutrition Alliance (IUNA), a formal association of the nutrition departments at the University
College Cork, Trinity College Dublin and the University of Ulster at Coleraine. Completion of the survey comes at an opportune time given the recent political developments within the island of Ireland and the establishment, in December 1999, of the Food Safety Promotion Board (FSPB). The FSPB is one of six North/South implementation bodies set up following the Belfast (Good Friday) Agreement and has the functions of promotion of food safety, food safety (including nutrition) research, food alerts, surveillance of food-borne disease, and promotion of scientific co-operation between laboratory services on the island. The FSPB, therefore, has close functional relationships with the respective enforcement agencies, the Food Safety Authority of Ireland and the more recently established Food Standards Agency in the UK. This initiative comes at the same time as the publication of the CEC White Paper on Food Safety (COM (1999) 719 final) that proposes the establishment of an independent European Food Authority as the most appropriate response to the need to guarantee a high level of food safety within the European Union (EU). The IUNA, through data emanating from the NSIFCS, is well placed to provide inputs into the work of the FSPB and the proposed European Food Authority.

The primary objective of the NSIFCS project was to establish an electronic database of the habitual food and drink consumption of Irish adults, aged 18-64 years, for (1) determination of national patterns of food and nutrient intakes, and (2) measurement of the intake of food chemicals, including pesticides, veterinary drug residues, contaminants, packaging materials and food additives. A secondary objective of the survey was investigation of the relationships between food and nutrient intake and factors such as health, lifestyle, socio-demographic variables, levels of physical activity, anthropometry, attitudes to diet and health, and restrained eating.

In fulfilling these objectives, the NSIFCS has provided information to facilitate the mandatory (additives and pesticides) and non-mandatory (packaging materials and contaminants) monitoring of food chemical intakes under EU Directives. These data can be used not only to protect 
consumers but also to set conditions of use of additives and pesticides.

The survey has also provided much needed up-to-date information on food and nutrient intake for use in nutrition policy and planning by health professionals. EURODIET $(2000)^{5}$ has identified, in drafts of working parties on Nutrition \& Diet for Healthy Lifestyles in Europe, that a barrier to the development of a coherent and comprehensive nutritional policy for the $\mathrm{EU}$, or even to adequate assessment of the impact of current policies and actions, is the lack of comparable data relating to the nutrient intakes, diets and lifestyles across Europe. Moreover, the lack of such comparable data inhibits the production of rational, evidence-based dietary guidelines.

Other important uses of the NSIFCS database are pertinent to the food industry, which can use the findings in food product promotion and food product development to ensure safety of consumers with respect to food chemical intake and to facilitate the attainment of nutrient targets and food-based dietary guidelines by consumers.

The protocol for the study obtained ethical approval from the Federated Dublin Voluntary Hospitals and St. James's Hospital Joint Research Ethics Committee in the Republic of Ireland and from the Research Ethical Committee of the University of Ulster in Northern Ireland. Funding was obtained from the Department of Agriculture, Food and Rural Development, Dublin, the Food Safety Authority of Ireland for data collection in the Republic of Ireland, and from an industrial consortium of partners including Bord Bia, Cadburys Ireland, Coca-Cola, Dairy Council for Northern Ireland, Kellogg's, Kerry Group, Mars Confectionery, Meat and Livestock Commission UK, National Dairy Council, Nestlé Ireland, Irish
Sugar, Tesco Ireland and the Irish Business and Employers' Confederation (IBEC) to cover work in the Republic of Ireland and in Northern Ireland.

The study commenced in 1997 and the first six months were spent consulting with international experts, setting up the methodology, training the research staff and designing the computer systems. A feasibility study was carried out in the Republic of Ireland from May to July 1997 in order to evaluate the proposed methods and to estimate response rates. The main phase of the fieldwork started in October 1997 and data collection was completed in October 1999. Details of the sampling, methodology and major findings from the database, together with conclusions and recommendations, are described in the following papers.

JJ Strain

Northern Ireland Centre for Diet and Health (NICHE), University of Ulster, Coleraine,

BT52 1SA, Northern Ireland

\section{References}

1 The Irish National Nutrition Survey. Proc. Nutr. Soc. 1950; 4: 269-95.

2 Lee P, Cunningham K. The Irish National Nutrition Survey. Dublin: Irish Nutrition and Dietetic Institute, 1990.

3 Barker ME, McClean SI, McKenna PG, Reid NG, Strain JJ, Thompson KA, Williamson AP, Wright ME. Diet, Lifestyle and Health in Northern Ireland. Coleraine: University of Ulster, 1988.

4 Gregory J, Foster K, Tyler H, Wiseman M. The Dietary and Nutritional Survey of British Adults. London: HMSO, 1990.

5 EURODIET (2000) [Online]. Available at http://eurodiet. med.uoc.gr/. 